\title{
Revealing the environs of the remarkable southern hot core G327.3-0.6
}

\author{
F. Wyrowski ${ }^{1}$, K. M. Menten ${ }^{1}$, P. Schilke ${ }^{1}$, S. Thorwirth ${ }^{1}$, R. Güsten ${ }^{1}$, and P. Bergman ${ }^{2,3}$ \\ 1 Max-Planck-Institut für Radioastronomie, Auf dem Hügel 69, 53121 Bonn, Germany \\ e-mail: wyrowski@mpifr-bonn.mpg.de \\ 2 European Southern Observatory, Alonso de Cordova 3107, Vitacura Casilla 19001, Santiago 19, Chile \\ 3 Onsala Space Observatory, Chalmers University of Technology, 43992 Onsala, Sweden
}

Received 3 April 2006 / Accepted 9 May 2006

\section{ABSTRACT}

\begin{abstract}
Aims. We present a submm study of the massive hot core G327.3-0.6 that constrains its physical parameters and environment. Methods. The APEX telescope was used to image $\mathrm{CO}$ and $\mathrm{N}_{2} \mathrm{H}^{+}$emission, to observe lines from other molecules toward a hot and a cold molecular core, and to measure the continuum flux density of the hot core.

Results. In the $\mathrm{C}^{18} \mathrm{O} J=3-2$ line, two clumps were found, one associated with the HII region G327.3-0.5 and the other associated with the hot core. An additional cold clump is found $30^{\prime \prime}(0.4 \mathrm{pc})$ northeast of the hot core in bright $\mathrm{N}_{2} \mathrm{H}^{+}$emission. From the the continuum data, we calculate a mass of $420 M_{\odot}$ and a size of $0.1 \mathrm{pc}$ for the hot core. A new, more accurate position of the hot core is reported, which allows the association of the core with a bright mid-infrared source. The luminosity of the hot core is estimated to be between 5 and $15 \times 10^{4} L_{\odot}$.

Conclusions. This study revealed several different evolutionary stages of massive star formation in the G327.3-0.6 region.
\end{abstract}

Key words. ISM: individual objects: G327.3-0.6 - ISM: clouds - stars: formation - radio lines: ISM - submillimeter

\section{Introduction}

There is no generally accepted evolutionary scheme for highmass star formation yet, in contrast to the detailed framework of CLASSes that exists for the early evolution of low-mass stars. Observationally, hot molecular cores represent an early evolutionary stage during the formation of massive stars. One of the most prominent hot molecular cores in the southern celestial hemisphere is the core associated with the HII region G327.3-0.6 at a kinematical distance of $2.9 \mathrm{kpc}$ (Bergman 1992). It is associated with prominent $\mathrm{H}_{2} \mathrm{O}, \mathrm{OH}$, and $\mathrm{CH}_{3} \mathrm{OH}$ masers, and its chemistry has been studied in two papers, one reporting ethylene oxide and acetaldehyde observations (Nummelin et al. 1998), while the other investigates the chemical inventory of this source (Gibb et al. 2000). The source is remarkable for its exceptionally rich molecular line spectra with relatively narrow, well-behaved (Gaussian) line profiles. This reduces line blending and makes them easier to interpret than spectra of many other very line-rich sources, such as Sgr B2. It is therefore surprising that almost nothing is known about its environs. Only Bergman (1992) reports some SEST maps that reveal two adjacent dense cores in this molecular cloud: one relatively cold ( $\left.T_{\text {kin }} \sim 30 \mathrm{~K}\right)$ cloud core and one hot $(T=100-200 \mathrm{~K})$ core. Hence, this region offers the possibility to study cores that have formed from the same parental cloud, but that are in different stages of evolution.

Since the G327.3-0.6 hot core has the potential of becoming a southern hemisphere hot core template for upcoming observatories like ALMA, we revisited this source and its environment with the recently commissioned APEX telescope (see Güsten et al., this volume).

\section{Observations}

The observations were done with the Atacama Pathfinder Experiment $\left(\mathrm{APEX}^{1}\right)$. The frontends used were the facility $345 \mathrm{GHz}$ and the MPIfR dual channel (460 and $810 \mathrm{GHz}$ ) FLASH receiver (Risacher et al. 2006; Heyminck et al. 2006, this volume). As backends, the MPIfR pocket backend (PBE) for the continuum and the MPIfR Fast Fourier Transform Spectrometer (FFTS, Klein et al. 2006, this volume) for the line observations were used. With the $345 \mathrm{GHz}$ receiver, several arcmin ${ }^{2}$-sized maps of ${ }^{12} \mathrm{CO}$ and $\mathrm{C}^{18} \mathrm{O}(3-2)$ were observed using the On-The-Fly (OTF) observing technique. $\mathrm{N}_{2} \mathrm{H}^{+}(3-2)$ was observed with raster mapping and the (4-3) and (5-4) transitions with single pointings towards the peak of the (3-2) emission. All observations were done in position switching mode. The continuum observations with the $345 \mathrm{GHz}$ and FLASH receivers were done as cross scans over the source using the continuum PBE. We used the hot core position $\left(\alpha_{\mathrm{J} 2000}, \delta_{\mathrm{J} 2000}\right)=(15: 53: 08.78,-54: 37: 01.20)$ from Bergman (1992) as the center of the maps.

\section{Results}

\subsection{CO mapping}

The large CO (3-2) map in Fig. 1 shows widespread $J=$ 3-2 emission. The strongest emission, indicating elevated

1 This publication is based on data acquired with the Atacama Pathfinder Experiment (APEX). APEX is a collaboration between the Max-Planck-Institut für Radioastronomie, the European Southern Observatory, and the Onsala Space Observatory. 


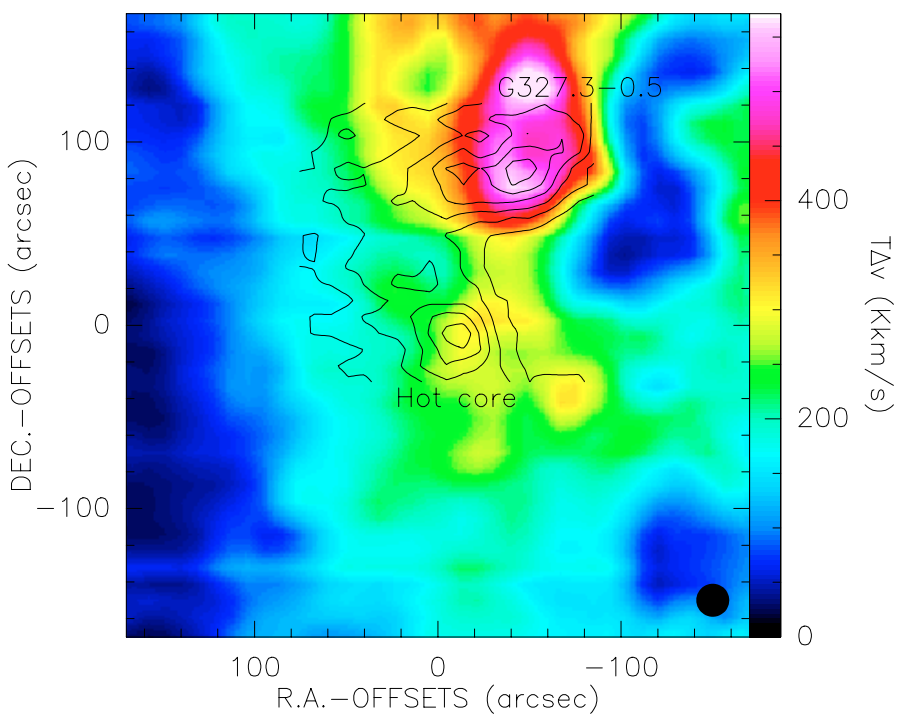

Fig. 1. APEX ${ }^{12} \mathrm{CO}$ (color) and $\mathrm{C}^{18} \mathrm{O}(3-2)$ (contours) images. The contour levels start at 23.6 with steps of $15.6 \mathrm{~K} \mathrm{~km} \mathrm{~s}^{-1}$.

temperatures in this optically thick line, is found in the north, and is associated with the strong HII region G327.3-0.5 (Goss $\&$ Shaver 1970) and a complex of infrared sources around IRS3 (Epchtein \& Lepine 1981). Lines in this region have temperatures going up to $70 \mathrm{~K}$, with widths of $10 \mathrm{~km} \mathrm{~s}^{-1}$ and typical velocities of $-48 \mathrm{~km} \mathrm{~s}^{-1}$. This $\mathrm{CO}$ emission likely traces the hot surface of a Photon Dominated Region (PDR) around G327.3-0.5.

There is an extension in the $\mathrm{CO}$ emission to the south where the hot core is located, but it is weaker by a factor of 2. Typical line temperatures and velocities in this region are $30 \mathrm{~K}$ and $-46 \mathrm{~km} \mathrm{~s}^{-1}$, respectively. Close, but offset from the hot core, the line profiles show weak evidence for outflow motions.

To the east, the $\mathrm{CO}$ emission vanishes rapidly, and to the west of the strong northern peak, it shows a conspicuous hourglass shaped "hole" where the CO emission is strongly reduced. This region might have been excavated by strong winds of an older generation of luminous stars, although with a SIMBAD search within $1^{\prime}$, no objects were found towards the center of the two evacuated lobes. In the GLIMPSE images, shell-like structures are found around the holes, as is expected for swept up material around stellar wind/radation cavities (Churchwell et al. 2004). Another less striking hole in CO and MIR emission is at $\left(5^{\prime \prime}, 140^{\prime \prime}\right)$.

Toward the northern peak and the hot core in the south, the line profiles show self absorption hinting toward a more complicated structure/layering with excitation gradients in the column density peaks seen in $\mathrm{C}^{18} \mathrm{O}$ (contours in Fig. 1). The hot core is clearly identified as a $\mathrm{C}^{18} \mathrm{O}$ peak, as is a ridge of strong $\mathrm{C}^{18} \mathrm{O}$ emission associated with the HII region G327.3-0.5. The peak within the ridge is at the position of one of the two strong ${ }^{12} \mathrm{CO}(3-2)$ emission maxima. Using the $\mathrm{C}^{18} \mathrm{O}(1-0)$ and $(2-1)$ line intensities, as measured by Bergman (1992), together with the new (3-2) measurement, we determine a column density of $6 \times 10^{16} \mathrm{~cm}^{-2}$ and an excitation temperature of $50 \mathrm{~K}$, which is somewhat higher than the temperature measured in ${ }^{12} \mathrm{CO}$, and is likely to be caused by a combination of increasing temperature to the inner part of the hot core and clumping within the beam. Using a $\mathrm{C}^{18} \mathrm{O}$ abundance of $2 \times 10^{-7}$ (Frerking et al. 1982), the mass in the beam is then $510 M_{\odot}$. With a size of $25^{\prime \prime}$ (after subtracting the large scale $\mathrm{C}^{18} \mathrm{O}$ emission) for the clump
Table 1. APEX continuum results.

\begin{tabular}{ccccc}
\hline \hline $\begin{array}{c}\text { Frequency } \\
(\mathrm{GHz})\end{array}$ & $\begin{array}{c}T_{\mathrm{MB}} \\
(\mathrm{K})\end{array}$ & $\begin{array}{c}\text { Widths } \\
\left({ }^{\prime \prime}\right)\end{array}$ & $\begin{array}{c}\text { Dec Widths } \\
\left({ }^{\prime \prime}\right)\end{array}$ & $\begin{array}{c}\text { Flux density } \\
(\mathrm{Jy})\end{array}$ \\
\hline 351 & 1.8 & 19.1 & 7.7 & 66 \\
464 & 3.0 & 14.8 & 6.6 & 117 \\
813 & 8.3 & 10.8 & 7.7 & 525 \\
\hline
\end{tabular}

harboring the hot core, a virial mass can be estimated to be $1330 M_{\odot}$, using the procedure described by Cesaroni et al. (1994).

\section{2. $\mathrm{CH}_{3} \mathrm{OH}$ results}

A strong methanol line was observed simultaneously with $\mathrm{C}^{18} \mathrm{O}$ (Table 2 and Fig. 3). The hot core is very prominent in methanol, but within the region mapped, it is the only methanol peak. The northern $\mathrm{C}^{18} \mathrm{O}$ peak was not detected in this molecule, which is surprising given its high temperatures and column densities. This might either be due to lower densities in the northern region so that the methanol transitions cannot be sufficiently excited or due to low methanol abundances in PDRs (Jansen et al. 1995). The peak of the emission is slightly offset by $5^{\prime \prime}$ from the one found by Bergman et al. (1992). The deconvolved size of the emission is $26 \times 7^{\prime \prime}$, with $\mathrm{PA}=60^{\circ}$ to the northeast.

\subsection{Continuum}

Continuum cross-scans of the hot core found the peak emission at $\left(-6^{\prime \prime},-6^{\prime \prime}\right)$, where the $\mathrm{CH}_{3} \mathrm{OH}$ also peaks. This is also consistent with the maps of complex molecules observed by Gibb et al. (2000). Fit results from averaged cross-scans at different frequencies are given in Table 1. The deconvolved size of the dust emission of the hot core is $7^{\prime \prime}$ or $0.1 \mathrm{pc}$, and is consistent at all three frequencies. The submm flux density measured towards the hot core is almost entirely due to thermal dust emission and allows a mass estimate of the hot core. Using the flux density at $850 \mu \mathrm{m}$, a dust emissivity of 1.8 from Ossenkopf \& Henning (1994, model 5), and a temperature of $100 \mathrm{~K}$ results in $420 M_{\odot}$ for the core. For a Hildebrand (1983) opacity with $\beta=2$, the mass is $950 M_{\odot}$.

\subsection{Spectral energy distribution}

With the improved position from our $\mathrm{CH}_{3} \mathrm{OH}$ and continuum observations, a clear association of the hot core with GLIMPSE point sources becomes possible (see Fig. 3). At the position of the hot core, a bright $8 \mu \mathrm{m}$ source is found that obeys the color and brightness criteria suggested by Ellingsen (2006) for massive star-forming cores harboring methanol masers. Only a few arcseconds off, another weaker source with high extinction is found, likely embedded in the hot core as well. In Fig. 2, the spectral energy distribution is shown towards the hot core. Additional millimeter flux densities are taken from Bergman (1992). Brand et al. (1984) estimate a $1.4 \mathrm{GHz}$ flux of $1 \mathrm{Jy}$ of what they call the southern extension of the HII region G326.3-0.5. This free-free flux and more extended dust emission might contribute to the excess emission seen in the $45^{\prime \prime}$ SEST beam at $3 \mathrm{~mm}$. Far infrared data points are the most important means of constraining the total luminosity of embedded young stellar objects. G327.3-0.6 itself is not associated with an IRAS point source, likely due to confusion or blending with the strong source IRAS 15492-5426. We checked 
Table 2. Line parameters towards molecular cores in the G327 region. For $\mathrm{N}_{2} \mathrm{H}^{+} T_{\text {peak }}$ was determined from Gaussian fits and the line widths from simultaneously fitting of all HFS components assuming the same excitation for all them, resulting in optical depths between 1-2. We also accounted for the blend of the $\mathrm{H}_{2} \mathrm{CO} 4_{32}-3_{31}$ and $4_{31}-3_{30}$ lines.

\begin{tabular}{|c|c|c|c|c|c|c|c|c|}
\hline$\overline{\text { Source }}$ & Transition & $\begin{array}{c}\text { Frequency } \\
(\mathrm{MHz})\end{array}$ & $\begin{array}{c}E_{\text {lower }} \\
(\mathrm{K})\end{array}$ & $\begin{array}{c}\text { Offsets } \\
\left({ }^{\prime \prime},{ }^{\prime}\right)\end{array}$ & $\begin{array}{c}\text { Size } \\
\left({ }^{\prime \prime}\right)\end{array}$ & $\begin{array}{c}T_{\text {peak }} \\
(\mathrm{K})\end{array}$ & $\begin{array}{c}\Delta v \\
\left(\mathrm{~km} \mathrm{~s}^{-1}\right)\end{array}$ & $\begin{array}{c}v_{\mathrm{LSR}} \\
\left(\mathrm{km} \mathrm{s}^{-1}\right)\end{array}$ \\
\hline North core & $\mathrm{C}^{18} \mathrm{O} 3-2$ & 329330.600 & 15.8 & $(-44,82)$ & & $21.3(0.7)$ & $4.9(0.1)$ & $-47.5(0.1)$ \\
\hline \multirow[t]{3}{*}{ Hot core } & $\mathrm{C}^{18} \mathrm{O} 3-2$ & & & $(-6,-6)$ & 25 & $15.8(1.0)$ & $6.2(0.2)$ & $-44.4(0.1)$ \\
\hline & $\mathrm{CH}_{3} \mathrm{OH} 7_{43}-6_{43}$ & 341415.625 & 63.7 & & $26 \times 7$ & $9.3(0.8)$ & $6.6(0.6)$ & $-45.3(0.2)$ \\
\hline & $\mathrm{N}_{2} \mathrm{H}^{+} 3-2$ & 279511.701 & 13.4 & & & $7.9(0.2)$ & $6.0(1.5)$ & $-45.3(0.3)$ \\
\hline \multirow[t]{5}{*}{ Cold core } & $\mathrm{N}_{2} \mathrm{H}^{+} 3-2$ & & & $(12,6)$ & $77 \times 39$ & $12.2(0.2)$ & $4.8(0.2)$ & $-45.9(0.1)$ \\
\hline & $\mathrm{N}_{2} \mathrm{H}^{+} 4-3$ & 372672.509 & 26.8 & $(12,6)$ & & $11.4(0.2)$ & $4.3(0.1)$ & $-45.8(0.1)$ \\
\hline & $\mathrm{N}_{2} \mathrm{H}^{+} 5-4$ & 465824.947 & 44.7 & $(12,6)$ & & $9.0(0.4)$ & $4.4(0.2)$ & $-45.4(0.1)$ \\
\hline & $\mathrm{H}_{2} \mathrm{CO} 4_{23}-3_{22}$ & 291237.781 & 68.1 & $(12,6)$ & & $2.3(0.2)$ & $4.5(0.2)$ & $-45.5(0.1)$ \\
\hline & $\mathrm{H}_{2} \mathrm{CO} 4_{32}-3_{31}$ & 291380.500 & 127.0 & $(12,6)$ & & $1.3(0.2)$ & $7.7(0.4)$ & $-45.2(0.2)$ \\
\hline
\end{tabular}

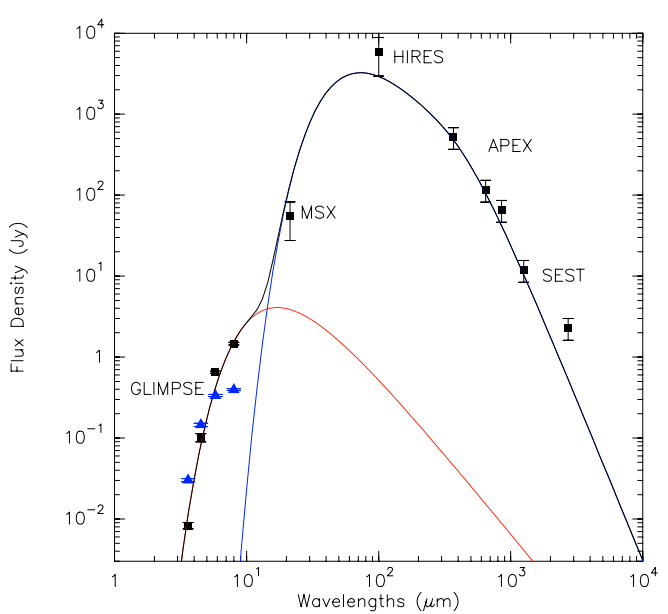

Fig. 2. Spectral energy distribution of the G327.3-0.6 hot core fit with a 2-component greybody fit. GLIMPSE fluxes of the source embedded in the cold core are marked as triangles.

$100 \mu \mathrm{m}$ HIRES images of the source and found that it breaks up into two sources; one is close in position to the hot core, and the flux of this source is given in Fig. 2. Also, the hot core is not associated with any MSX source. The closest MSX source is the extended feature $10^{\prime \prime}$ to the west of the hot core, we therefore estimated the $20 \mu \mathrm{m}$ MSX flux of the hot core directly from MSX images. We take the high uncertainty in the 20 and $100 \mu \mathrm{m}$ fluxes, due to the confusion with other sources with $50 \%$ error bars, into account. In Fig. 2, a simple 2-component fit of all the flux densities is shown, with a greybody at $70 \mathrm{~K}$ fitting the $\mathrm{mm} / \mathrm{submm} /$ FIR part and a blackbody at $300 \mathrm{~K}$ fitting the MIR emission of the GLIMPSE source. Given the uncertainties of the FIR fluxes, we estimate a luminosity between $5-15 \times 10^{4} L_{\odot}$ for the hot molecular core.

\section{5. $\mathrm{N}_{2} \mathrm{H}^{+}$mapping}

Figure 3 shows the $8 \mu \mathrm{m}$ GLIMPSE image of the region with contours of $\mathrm{CH}_{3} \mathrm{OH}$ and $\mathrm{N}_{2} \mathrm{H}^{+}(3-2)$ overlaid. The hot core seen in methanol is situated at the edge of an arcmin scale midinfrared dark cloud, which is traced well in $\mathrm{N}_{2} \mathrm{H}^{+}$. The $\mathrm{N}_{2} \mathrm{H}^{+}$ peak seems to trace a colder clump in an earlier evolutionary state. Interestingly, even the cold clump already harbors a hotter component, as is evident from the simultaneously detected $\mathrm{H}_{2} \mathrm{CO}$ lines (Fig. 4) and the highly reddened GLIMPSE sources (blue markers in Fig. 2). The total size of the $\mathrm{N}_{2} \mathrm{H}^{+}$clump is $1 \mathrm{pc}$.

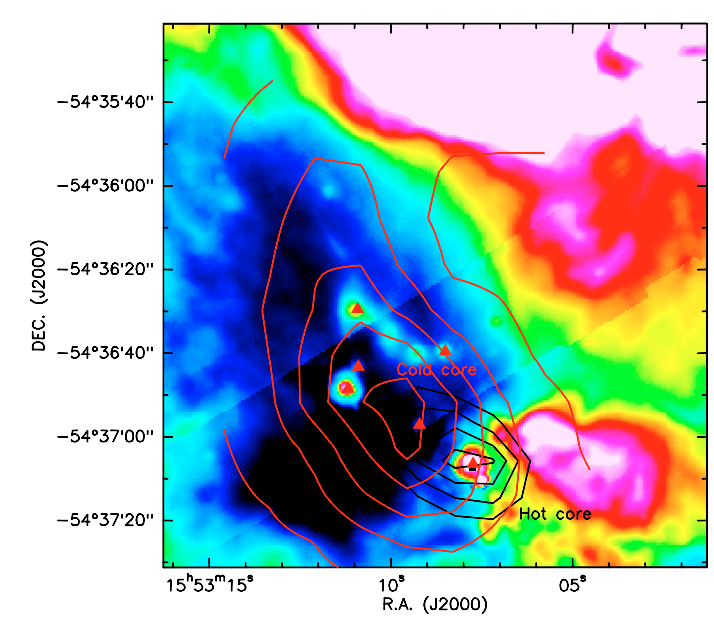

Fig. 3. APEX images in the $\mathrm{N}_{2} \mathrm{H}^{+}(3-2)$ and the $\mathrm{CH}_{3} \mathrm{OH} 7_{43}-6_{43}$ lines (red and black contours) overlaid on the $8 \mu \mathrm{m}$ GLIMPSE emission. Embedded GLIMPSE point sources with massive YSO characteristics are marked with triangles.

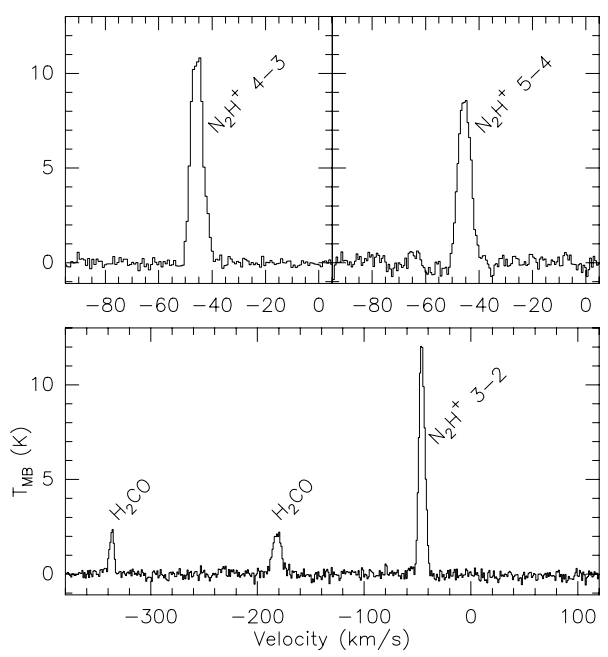

Fig. 4. APEX $\mathrm{N}_{2} \mathrm{H}^{+}$spectra observed toward the cold core at (12", $\left.6^{\prime \prime}\right)$.

The high and comparable peak temperatures of the 3 observed $\mathrm{N}_{2} \mathrm{H}^{+}$lines (see Table 2) suggest that they are optically thick. We used RADEX on-line ${ }^{2}$ to investigate the required excitation conditions for the observed intensities. Similar excitation temperatures for both lines can only be reached for densities $>10^{6} \mathrm{~cm}^{-3}$ unless the $\mathrm{N}_{2} \mathrm{H}^{+}$abundances

${ }^{2}$ http://www.strw.leidenuniv.nl/ moldata/radex.php 
are higher than $3 \times 10^{-9}$. With a kinetic temperature of $18 \mathrm{~K}$, the line intensities can be reproduced, but still require $\mathrm{H}_{2}$ column densities of the order of $10^{24} \mathrm{~cm}^{-2}$. The high column density, together with weak $\mathrm{C}^{18} \mathrm{O}$, suggests that $\mathrm{C}^{18} \mathrm{O}$ is depleted in the cold clump, and maybe even in the outer envelope of the hot core clump, which would explain the discrepancy between $\mathrm{C}^{18} \mathrm{O}$ and virial mass. Using Cesaroni et al. (1994) again, the virial mass of the $60^{\prime \prime} \mathrm{N}_{2} \mathrm{H}^{+}$clump is $1000 M_{\odot}$.

\section{Conclusions}

A detailed view onto the environs of G327.3-0.6 is another example of the complexity of massive star-forming regions, since many observational phenomena occur simultaneously and can only be separated with adequate angular resolution. In what we think is the order of decreasing evolutionary stages, the phenomena in this region are:

- suspicious holes in the CO emission possibly excavated by B-star winds;

- the bright HII region G327.3-0.5 to the north associated with a luminous PDR seen in CO;

- an ultracompact HII region 2 arcmin $(1.7$ pc) south, seen as a southern extensions of the old $\mathrm{cm}$ continuum maps and as bright extended MIR emission by GLIMPSE;

- 5-10 arcsec $(0.07-0.14 \mathrm{pc})$ offset from the UC HII region, the hot molecular core harboring several MIR point sources; and
- further east of the hot core, a dense and cold clump with an embedded massive young stellar object.

The mass and luminosity of the hot core of about $500 M_{\odot}$ and $10^{5} L_{\odot}$ are comparable to "classical" luminous hot cores like G10.47+0.03 and G31.41+0.31 (Hatchell et al. 2000), but for further studies the advantage of G327.3-0.6 compared to those regions will be that its distance is a factor of 2 smaller.

\section{References}

Brand, J., van der Bij, M. D. P., de Vries, C. P., et al. 1984, A\&A, 139, 181 Bergman, P. 1992, Ph.D. Thesis, Göteborg

Cesaroni, R., Churchwell, E., Hofner, P., Walmsley, C. M., \& Kurtz, S. 1994, A\&A, 288, 903

Churchwell, E., Whitney, B. A., Babler, B. L., et al. 2004, ApJS, 154, 322

Ellingsen, S. P. 2006, ApJ, 638, 241

Epchtein, N., \& Lepine, J. R. D. 1981, A\&A, 99, 210

Frerking, M. A., Langer, W. D., \& Wilson, R. W. 1982, ApJ, 262, 590

Gibb, E., Nummelin, A., Irvine, W. M., Whittet, D. C. B., \& Bergman, P. 2000, ApJ, 545, 309

Goss, W. M., \& Shaver, P. A. 1970, Austr. J. Phys. Astrophys. Suppl., 14, 1

Hatchell, J., Fuller, G. A., Millar, T. J., Thompson, M. A., \& Macdonald, G. H. 2000, A\&A, 357, 637

Hildebrand, R. H. 1983, QJRAS, 24, 267

Jansen, D. J., van Dishoeck, E. F., Black, J. H., Spaans, M., \& Sosin, C. 1995, A\&A, 302, 223

Nummelin, A., Dickens, J. E., Bergman, P., et al. 1998, A\&A, 337, 27

Ossenkopf, V., \& Henning, T. 1994, A\&A, 291, 943 